\title{
Development of a Sustainable Catalytic Ester
}

\section{Amidation Process}

\author{
Nicola Caldwell,,$^{\dagger}$ Craig Jamieson, ${ }^{*}{ }^{\dagger}$ Iain Simpson ${ }^{\ddagger}$ and Allan J. B. Watson ${ }^{\dagger}$ \\ ${ }^{\dagger}$ Department of Pure and Applied Chemistry, WestCHEM, University of Strathclyde, 295 \\ Cathedral Street, Glasgow, G1 1XL, UK; ${ }^{\ddagger}$ AstraZeneca, Oncology Innovative Medicines Unit, \\ Mereside, Alderley Park, Macclesfield, SK10 4TG, UK; *Email: craig.jamieson@strath.ac.uk
}

KEY WORDS: Amidation, Green Solvents, Replacement Bases, Reaction Screening, Design of Experiments.

ABSTRACT: We describe the development of a sustainable ester amidation process. Base and solvent screening, combined with the application of Design of Experiments methodology was employed to identify an optimized set of reaction conditions using a sustainable protocol. Utilizing these optimized conditions, treatment of a range of ester derivatives with amino alcohols in the presence of a catalytic quantity of an $\mathrm{K}_{3} \mathrm{PO}_{4}$ deploying iso-propanol as solvent results in the highly efficient generation of a range of amido-alcohol derivatives in good to excellent yield, accompanied with excellent Reaction Mass Efficiency (RME).

\section{INTRODUCTION}

From consideration of both biology and synthesis, the amide functional group is a ubiquitously encountered motif. ${ }^{1,2}$ It represents the fundamental linking unit in peptides and proteins and is 
frequently encountered in small molecules, particularly in a pharmaceutical setting. Accordingly, considerable efforts have been, and continue to be, invested in the synthesis of amide-containing molecules. A number of recent reviews ${ }^{3-5}$ have underlined the frequency at which amide bond formation is executed in Medicinal Chemistry laboratories, with this single reaction accounting for a significant fraction of all transformations performed in this setting. Consequently, reliable methods for the synthesis of the amide bond are keenly sought. Preeminent among these approaches are use of stoichiometric reagents to facilitate the formal condensation of an acid and an amine. ${ }^{6}$

Despite the wide utility of this approach, and as intimated by Alfonsi ${ }^{7}$ and co-workers at Pfizer, this overall strategy of using stoichiometric reagents is tainted with a number of issues from a green chemistry perspective. Notably, uronium salts such as $\mathrm{HATU}^{8}$ have significant byproducts which are disproportionate in relation to the overall transformation being carried out, offering very low atom economy and associated reaction mass efficiencies. ${ }^{9}$ Based on this, in recent years there have been concerted efforts made to develop catalytic approaches to amide condensations. ${ }^{10-15}$ Such methodology offers greater reaction efficiency metrics, together with minimising environmental impact.

To this end, we recently reported a base-catalysed process for the conversion of unactivated ester derivatives to amido-alcohol adducts through reaction with amino alcohol derivatives (Figure 1). ${ }^{16}$ The reaction proceeds through an initial transesterification event which is mediated by exogenous base, followed by rearrangement to the thermodynamically more desirable amide product. This reaction manifold was demonstrated to be of wide utility and of relevance to the preparation of biologically active compounds. Having stated this, and based on consideration of the principles enunciated by Alfonsi on the development of green chemistry 
tools, ${ }^{7}$ we reasoned there was scope to address both the green credentials and scalability of the process to furnish a reaction with the optimum balance of utility, greenness, and fitness for scaleup. In this paper, and as part of our ongoing interests in the advancement and uptake of green synthesis in a Discovery Chemistry setting, ${ }^{17-19}$ we report our efforts to develop a significantly more sustainable process and demonstrate its applicability to the synthesis of a range of lead-like substrates.

\section{EXPERIMENTAL}

General Experimental Procedure A for Initial Base and Solvent Screening

To an oven-dried Schlenk tube containing the appropriate base $(0.28 \mathrm{mmol}, 0.2$ equiv) and solvent $(1.4 \mathrm{~mL}, 1 \mathrm{M})$ was added methyl benzoate, $(178 \mu \mathrm{L}, 1.42 \mathrm{mmol}, 1$ equiv) and ethanolamine ( $86 \mu \mathrm{L}, 1.42 \mathrm{mmol}, 1$ equiv). The reaction mixture was stirred at $40{ }^{\circ} \mathrm{C}$ for $22 \mathrm{~h}$. The reaction mixture was sampled at 4,8 and 22 hour time points and the conversion was determined by HPLC with reference to an internal standard. Full details are provided in the Supporting Information.

General Experimental Procedure B for Optimization of $\mathrm{K}_{3} \mathrm{PO}_{4}$-Catalysed Reaction

To an oven-dried Schlenk tube containing $\mathrm{K}_{3} \mathrm{PO}_{4}(0.1-0.3$ equiv) and $i$-PrOH $(0.5-2 \mathrm{M})$ was added methyl benzoate, (178 $\mu \mathrm{L}, 1.42 \mathrm{mmol}, 1$ equiv) and ethanolamine ( $86 \mu \mathrm{L}, 1.42 \mathrm{mmol}, 1$ equiv). The reaction mixture was stirred at the required temperature $\left(20-60{ }^{\circ} \mathrm{C}\right)$ for $8-22$ hours. The reaction mixture was sampled at the end of the required reaction time and the conversion was determined by HPLC with reference to an internal standard. Refer to the Supporting Information for full details. 
General Experimental Procedure C for the Synthesis of Amido Alcohols via Base-Catalysed Amide Bond Formation

For example: Compound 3. N-(2-hydroxyethyl)benzamide. To an oven-dried Schlenk tube containing $\mathrm{K}_{3} \mathrm{PO}_{4}(90 \mathrm{mg}, 0.43 \mathrm{mmol}, 0.3$ equiv) and $i$-PrOH $(700 \mu \mathrm{L})$ was added methyl benzoate (178 $\mu \mathrm{L}, 1.42 \mathrm{mmol}, 1$ equiv) and ethanolamine (86 $\mu \mathrm{L}, 1.42 \mathrm{mmol}, 1$ equiv). The reaction mixture was stirred at $60^{\circ} \mathrm{C}$ for $22 \mathrm{~h}$ then concentrated to a residue that was purified by silica solid phase extraction (5\% methanol/CPME) to afford the title compound (188 $\mathrm{mg}, 80 \%)$. Full characterization of this and all compounds synthesized is provided in the Supporting Information.

\section{RESULTS AND DISCUSSION}

In an effort to enhance the profile of the process shown in Figure 1 to achieve a balance of utility, sustainability and scalability we focused on two major considerations: (i) choice of base; and (ii) solvent selection. The condensation reaction had previously been enabled with the phosphazene base BEMP (2-tert-butylimino-2-diethylamino-1,3-dimethylperhydro-1,3,2diazaphosphorine). ${ }^{20}$ While this was effective and could be deployed catalytically, we sought to identify a more sustainable and cost-effective replacement.

In order to identify a suitable alternative catalyst, we embraced the rubric of Adams ${ }^{21}$ who utilized a scoring system based on health, safety and environmental risk phrases in constructing reagent guides designed to reduce impact to the environment in drug discovery and development. Accordingly, we made assessment of environment, health and safety scores using risk phrases to identify replacements for BEMP which also possessed an acceptable balance of 
practicality for use. Based on this, we elected to screen the inorganic bases $\mathrm{K}_{3} \mathrm{PO}_{4}$ and $\mathrm{K}_{2} \mathrm{CO}_{3}$, triethylamine and the carbonate mineral hydrotalcite. ${ }^{22}$ Additionally, we chose to screen the clayderived reagent montmorillonite as it had previously been shown to facilitate transesterification reactions ${ }^{23}$ hence could, in principle, catalyze the first step of our process.

The second aspect of our study centered around the solvent deployed in the condensation. Previous work by Constable ${ }^{24}$ has indicated that solvents constitute around $56 \%$ of the materials used to prepare an API, underlining the importance of this factor in designing a sustainable process. Accordingly, considerable effort has been invested towards the identification of more environmentally acceptable solvent systems, with a number of analyses appearing in the literature in recent years. ${ }^{7,25-27}$ While the existing process was not intensive in its use of solvent as it is carried out at relatively high $(2 \mathrm{M})$ concentration, we were mindful of the need to identify a replacement solvent. Indeed, recent analysis by Henderson $e a^{25}$ has suggested that there is an urgent need to replace acetonitrile with a more sustainable alternative. From consideration of this analysis, we selected either existing or emerging substitutes: 2-methyltetrahydrofuran (2MeTHF) ${ }^{28}$ iso-propyl alcohol (i-PrOH), cyclopentylmethyl ether (CPME), ${ }^{28-30}$ and tert-butyl methyl ether (TBME). ${ }^{31}$ Although there is limited evidence of a potential carcinogenic effect when using high quantities of TBME, ${ }^{32}$ only small amounts were used in this study and it was concluded that the sustainable properties outweighed the potential risks. Other potential solvents such as $\mathrm{MeOH}$ and acetone were discounted on the basis of possible competing side reactions occurring (transesterification and imine formation, respectively). 
Having made appropriate selections in relation to acceptable bases and solvents, we initiated our optimization campaign by screening combinations of solvent and base for their efficacy in a model reaction between methyl benzoate $\mathbf{1}$ and ethanolamine $\mathbf{2}$ to furnish the amide $\mathbf{3}$ (Figure 2).

Using the conditions and reagents outlined in Figure 2, conversions for each reaction were determined by HPLC and a temporal profile for each combination constructed. Based on this analysis, the most promising alternative base identified was $\mathrm{K}_{3} \mathrm{PO}_{4}$, which demonstrated appreciable conversion (ca. 35\%) to amide 3 in two of the replacement solvents (CPME and $i$ PrOH, Figure 3). Although there is potential for eutrophication associated with the phosphate species, ${ }^{33}$ the quantities used in the current setting are essentially negligible in comparison to, for example, that used in agriculture. Against this background, the scale at which the chemistry is carried out goes some way to allaying this specific concern, particularly given the reaction is catalytic in nature. The remaining two sustainable solvents exhibited comparatively lower conversions (ca. $15-20 \%$ ) and were therefore deemed less suitable as replacements for acetonitrile.

With appropriate replacement base catalyst and solvents in hand, we next turned our attention to optimizing the yield of the process shown in Fig. 2. In order to achieve this in an expedient manner which could simultaneously explore the effects of multiple parameters, we employed the technique of Design of Experiments. ${ }^{34}$ In this study, we used a two-level, half-fractional factorial design to probe the following variables: temperature $\left(20-60{ }^{\circ} \mathrm{C}\right)$, concentration $(0.5-2 \mathrm{M})$, reaction time $(8-22 \mathrm{~h})$ and catalyst loading $(10-30 \mathrm{~mol} \%)$. Table 1 indicates the specific set of conditions employed, including two centre points in the design to allow for estimation of error (Entries 4 and 8). Although CPME showed similar efficacy in the screening study, we elected to run the optimisation using $i$-PrOH as solvent based on the comparatively lower cost of the latter. 
Inspection of the dataset generated from the factorial design process suggested that good conversion to product 3 could be achieved when using elevated temperatures and $30 \mathrm{~mol} \%$ catalyst loading. Indeed, more detailed examination of the data using a response surface indicated that these two effects were the most important in terms of influencing conversion (Figure 4).

Having identified the most important parameters in controlling the overall reaction efficiency, we next evaluated the reaction of 1 and 2 using $30 \mathrm{~mol} \% \quad \mathrm{~K}_{3} \mathrm{PO}_{4}$ in $i-\mathrm{PrOH}(2 \mathrm{M})$ at $60{ }^{\circ} \mathrm{C}$ for $22 \mathrm{~h}$. Pleasingly, this furnished the target amide $\mathbf{3}$ in $80 \%$ isolated yield, representing a substantial enhancement in the efficiency of the process. Interestingly, increasing the reaction temperature to $80{ }^{\circ} \mathrm{C}$ did not prove to be more effective, with $71 \%$ conversion to 3 measured under these conditions. In this case, the bis-acylated ethanolamine derivative 4 (Fig. 5) could be isolated which may potentially account for the observed reduction in yield.

With optimal conditions using more sustainable reagents identified, we next focused on exemplifying the process on a range of diverse substrates (Table 2). In keeping with the optimisation phase of the programme, we sought to retain a 1:1 stoichiometry of ester to amino alcohol derivative in order to make efficient use of these building blocks and to offer enhanced Reaction Mass Efficiency (RME, vide infra). These substrates were selected in order to enable ready comparison with our first generation process ${ }^{16}$ enabling us to gauge the effectiveness of the current, more sustainable protocol. In any discovery chemistry setting, chromatographic purification of final compounds is usually required prior to undertaking a bioassay in order to have confidence in the data generated. We were cognizant of the significant contribution that eluents used in this process make to overall waste, so accordingly we also sought to modify the solvent system used in the purification process. Based on results recently generated in our 
laboratories, ${ }^{17}$ in the current study we were able to replace the $\mathrm{CH}_{2} \mathrm{Cl}_{2} / \mathrm{MeOH}$ eluent system used previously with the more environmentally acceptable $\mathrm{CPME} / \mathrm{MeOH}$ pairing (see Experimental Section and Supporting Information for further details).

We initially surveyed a number of aromatic derivatives (entries $1-4$ ) and established good to excellent yields with this substrate class using the conditions shown. Turning our attention to heteroaromatic ester derivatives (entries 5 -9), we again noted smooth conversion to the desired amide products with excellent isolated yields generally being observed. Also within this substrate class, we demonstrated that variation of the amino alcohol derivative was tolerated (entries 7, 8, and 9) with both propanolamine and $(S)$-2-pyrrolidinemethanol being more than competent substrates for our optimised process. Focusing on a third substrate class, we then demonstrated that alkyl ester derivatives proved to be similarly effective substrates (entries $10-$ 14) with isolated yields residing in the good to excellent range. In the final aspect of our exemplification study, we examined peptide derived substrates in our reaction system. Subjecting Boc-Gly-OMe and phenylalaninol to our optimised conditions furnished the dipeptoid 18 (entry 15) in good yield. Extending this approach to the more sterically demanding substrate Boc-Phe-OMe was successful in delivering the target dipeptoid 19 (entry 16), albeit in modest yield in comparison to 18 . Having stated this, product 19 could be prepared with negligible erosion of chiral integrity $(\mathrm{d} . \mathrm{r}=98: 2)$, indicating the potential utility of our method when using enantioenriched esters as substrates. Additionally, the measured diastereomeric ratio obtained with the sustainable process is superior to that obtained with the first generation protocol (d.r. $=$ 86:14), highlighting another potential advantage. Overall, consideration of the isolated yields obtained with this new and sustainable coupling protocol indicates that the current method is an extremely viable alternative compared to the previously developed approach. ${ }^{16}$ Indeed, 
examination of the average yield for this newly developed protocol compared with the previous methodology ( 84 and $86 \%$, respectively) again indicates the utility of the more sustainable approach.

In addition to isolated yields, we also sought to analyse the efficiency of the optimised reaction from a green chemistry perspective. To this end, we focused on the RME metric proposed by Curzons $e t a l^{9}$ which is not only an expression of yield but reflects the molar quantities of reactants, as well as the general ethos of atom economy. This analysis would then offer some means of quantifying how 'green' the overall process is. Appraisal of the data suggests that the RME values for the transformations in Table 2 are generally good (with the exception of entries 9 and 16). The mean RME value calculated by Curzons for a series of amide bond forming reactions was $62 \%{ }^{9}$ By comparison, the majority of the exemplars in Table 2 are in excess of this value, suggesting a much more efficient process compared to this earlier benchmark. This trend is reinforced upon calculation of the average RME within this dataset which reveals a value of $70 \%$, again demonstrating a highly favourable efficiency when compared to metrics available in the literature.

In the last phase of our study we sought to demonstrate the utility of the optimized reaction upon scale-up. Accordingly, we targeted the preparation of compounds $\mathbf{8}$ and $\mathbf{1 3}$ on a $10 \mathrm{mmol}$ scale. Gratifyingly, this provided gram-quantities of products in acceptable yields $(92 \%$ and $85 \%$, respectively), with calculated RME values again above the high watermark established by Curzons $^{9}$ ( $76 \%$ and $72 \%$ for $\mathbf{8}$ and $\mathbf{1 3}$, respectively). This initial study bodes well for adapting the optimized process on larger scales in a discovery chemistry setting.

In summary, through a combination of reaction screening and statistically driven design, we have developed a robust and green set of conditions for the conversion of unactivated ester 
derivatives to amide containing products using a base-mediated protocol. The reaction conditions have been applied to a range of products in generally good to excellent yields, accompanied by highly encouraging efficiency metrics from a sustainability perspective. In addition, the effectiveness of the reaction on scale has also been demonstrated. Based on all of the above, we believe that this new process will be of considerable utility in the preparation of lead and drug molecules in a sustainable manner. 


\section{FIGURES}

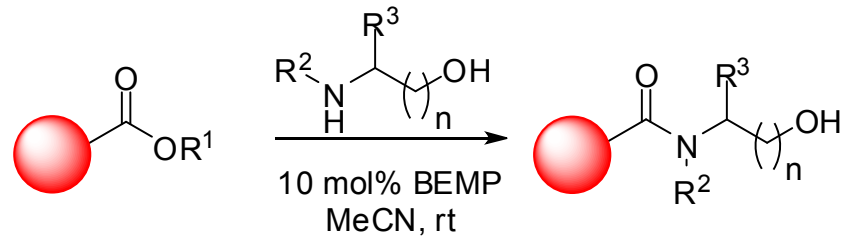

Figure 1. Base-mediated amidation of esters with amino alcohols.

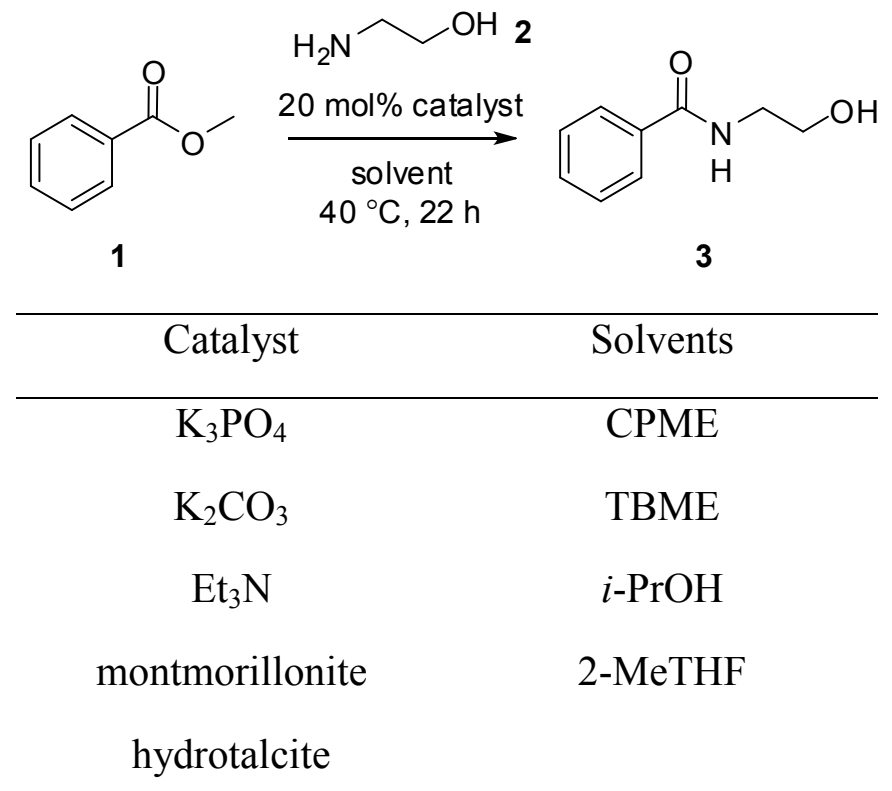

$\overline{\text { Figure 2. Screening of alternative solvents and bases. }}$

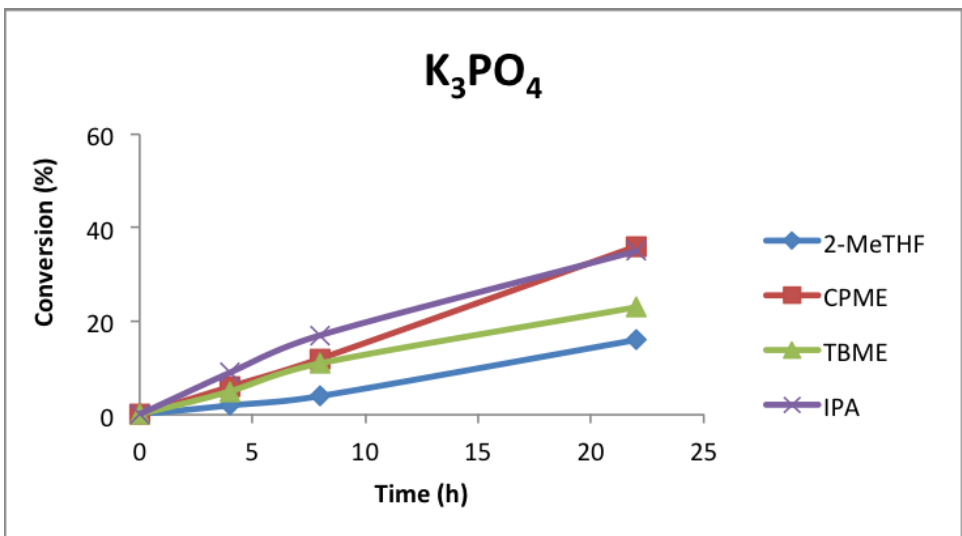

Figure 3. $\mathrm{K}_{3} \mathrm{PO}_{4}$ mediated conversion of $\mathbf{1}$ to amide 3 


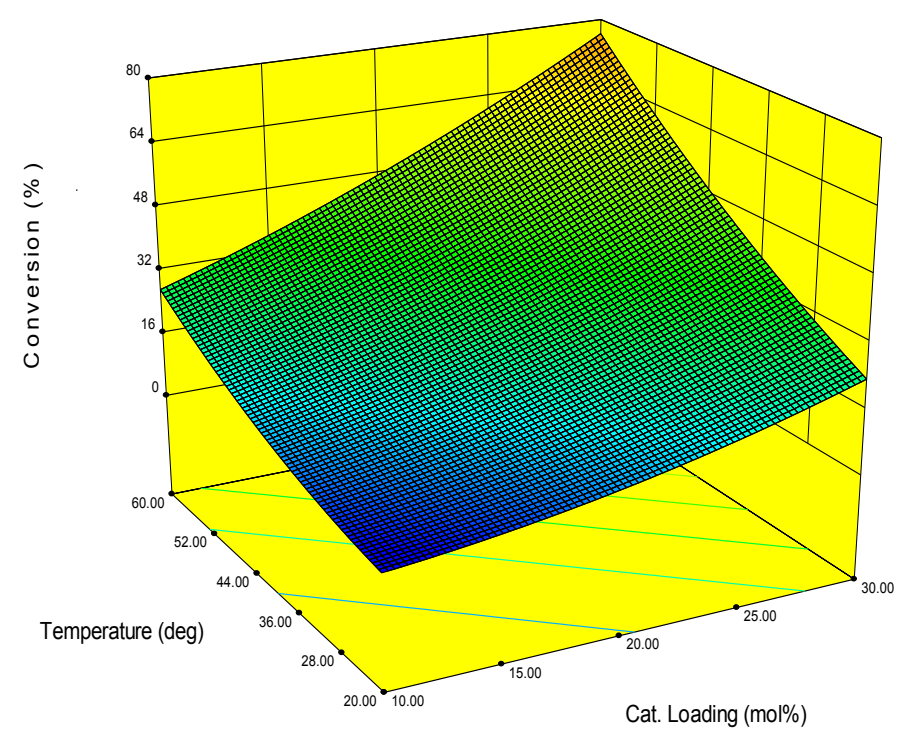

Figure 4. Response surface modelling the effects of catalyst loading and temperature for the conversion of 1 to 3 .<smiles>O=C(NCCOC(=O)c1ccccc1)c1ccccc1</smiles>

Figure 5. Bis-acylated ethanolamine by-product

Table 1. Factorial design for the optimisation of the conversion of $\mathbf{1}$ to $\mathbf{3}$.

\begin{tabular}{cccccc}
\hline Entry & Time $(\mathrm{h})$ & Temp $\left({ }^{\circ} \mathrm{C}\right)$ & Conc. $(\mathrm{M})$ & $\begin{array}{c}\text { Cat. Loading } \\
(\mathrm{mol} \%)\end{array}$ & Conversion $(\%)^{\mathrm{a}}$ \\
\hline 1 & 8 & 60 & 0.5 & 30 & 31 \\
2 & 22 & 60 & 0.5 & 10 & 17 \\
3 & 8 & 20 & 2 & 30 & 8 \\
4 & 15 & 40 & 1.25 & 20 & 19
\end{tabular}




\begin{tabular}{cccccc}
5 & 22 & 20 & 0.5 & 30 & 14 \\
6 & 8 & 60 & 2 & 10 & 37 \\
7 & 22 & 60 & 2 & 30 & 82 \\
8 & 15 & 40 & 1.25 & 20 & 20 \\
9 & 8 & 20 & 0.5 & 10 & 3 \\
10 & 22 & 20 & 2 & 10 & 5 \\
\hline
\end{tabular}

${ }^{a}$ Determined by HPLC using an internal standard. 
Table 2. Exemplification of substrate scope.

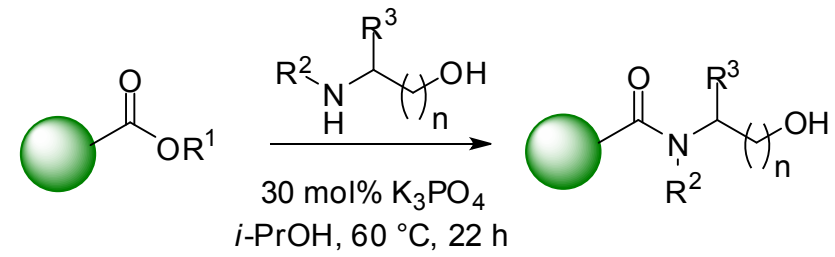

\begin{tabular}{llll}
\hline Entry & Yield (\%) & RME (\%) \\
\hline & & & \\
\end{tabular}




12 (10)

\footnotetext{
${ }^{a}$ Isolated yield. ${ }^{b}$ Figures in parenthesis represent isolated yields obtained from the progenitor methodology (Figure 1 and Ref. 16). ${ }^{c}$ Reaction Mass Efficiency. ${ }^{d}$ n.d. $=$ not determined.
}

\section{ASSOCIATED CONTENT}

Supporting Information available: Full experimental procedures and analytical data for all compounds. This material is available free of charge via the Internet at http://pubs.acs.org. 


\section{AUTHOR INFORMATION}

\section{Corresponding Author}

* Email: craig.jamieson@strath.ac.uk; Fax: +44 (0)141 548 4822; Tel: +44 (0)141 5484830

\section{Author Contributions}

The manuscript was written through contributions of all authors. All authors have given approval to the final version of the manuscript

\section{Notes}

The authors declare no competing financial interests.

\section{ACKNOWLEDGMENT}

We thank the EPSRC and AstraZeneca for financial support. Mass spectrometry data was acquired at the EPSRC UK National Mass Spectrometry Facility at Swansea University.

\footnotetext{
ABBREVIATIONS

API, active pharmaceutical ingredient; BEMP, 2-tert-butylimino-2-diethylamino-1,3dimethylperhydro-1,3,2-diazaphosphorine; CPME, cyclopentylmethyl ether; HATU, O-(7azabenzotriazol-1-yl)- $N, N, N^{\prime}, N^{\prime}$-tetramethyluronium hexafluorophosphate; RME, reaction mass efficiency; TBME, tert-butyl methyl ether.
} 


\section{REFERENCES}

1 Peptides, Synthesis, Structures and Applications; Gutte, B., Ed.; Academic Press: San Diego, 1995.

2 Pattabiraman, V. R and Bode, J. W., Rethinking Amide Bond Synthesis, Nature 2011, $480,471-479$.

3 Roughley, S. D. and Jordan, A. M., The Medicinal Chemist's Toolbox: An Analysis of Reactions Used in the Pursuit of Drug Candidates, J. Med. Chem. 2011, 54, 3451-3479.

4 Cooper, T. W. J.; Campbell, I. B; Macdonald, S. J. F., Factors Determining the Selection of Organic Reactions by Medicinal Chemists and the Use of These Reactions in Arrays (Small Focused Libraries), Angew. Chem. Int. Ed. 2010, 49, 8082-8091.

5 Carey, J. S.; Laffan, D.; Thomson, C.; Williams, M. T., Analysis of the Reactions Used for the Preparation of Drug Candidate Molecules, Org. Biomol. Chem. 2006, 4, 2337-2347.

6 For a review see: El-Faham, A. and Albericio, F., Peptide Coupling Reagents, More than a Letter Soup, Chem. Rev. 2011, 111, 6557-6602.

7 Alfonsi, K.; Colberg, J.; Dunn, P. J.; Fevig, T.; Jennings, S.; Johnson, T. A.; Kleine, H. P.; Knight, C.; Nagy, M .A.; Perry, D. A.; Stefaniak, M., Green Chemistry Tools to Influence a Medicinal Chemistry and Research Chemistry Based Organisation, Green Chem. 2008, 10, 3136.

8 Carpino, L. A. 1-Hydroxy-7-azabenzotriazole. An Efficient Peptide Coupling Additive , J. Am. Chem. Soc. 1993, 115, 4397-4398. 
9 Curzons, A. D.; Constable, D. J. C.; Mortimer, D. N.;. Cunningham, V. L., So You Think Your Process is Green, How Do You Know? - Using Principles of Sustainability to Determine What is Green - a Corporate Perspective, Green Chem. 2001, 3, 1-6.

10 Sabot, C.; Kumar, K. A.; Meunier, S.; Mioskowski, C., A Convenient Aminolysis of Esters Catalysed by 1,5,7-Triazabicyclo[4.4.0]dec-5-ene (TBD) Under Solvent-Free Conditions, Tetrahedron Lett. 2007, 48, 3863-3866.

11 Arnold, K.; Batsanov, A. S.; Davies, B. Whiting, A., Synthesis, Evaluation and Application of Novel Bifunctional N,N-Di-isopropylbenzylamineboronic Acid Catalysts for Direct Amide Formation Between Carboxylic Acids and Amines, Green Chem. 2008, 10, 124134.

12 Allen, C. L.; Chhatwal, C. R.; Williams, J. M. J., Direct Amide Formation From Unactivated Carboxylic Acids and Amines, Chem. Comm. 2012, 48, 666-668.

13 Ghosh, S.; Bhaumik, A.; Mondal, J.; Mallik, A.; Sengupta, S.; Mukhopuahyay, C., Direct Amide Bond Formation from Carboxylic Acids and Amines Using Activated Alumina Balls as a New, Convenient, Clean, Reusable and Low Cost Heterogeneous Catalyst, Green Chem. 2012, $14,3220-3229$.

14 Gernigon, N.; Al-Zoubi, R. M.; Hall, D. G., Direct Amidation of Carboxylic Acids Catalyzed by ortho-Iodo Arylboronic Acids: Catalyst Optimisation, Scope and Preliminary Mechanistic Study Supporting a Peculiar Halogen Acceleration Effect, J. Org. Chem. 2012, 77, 8386-8400. 
15 Ohshima, T. I.; Hayashi, Y.; Agura, K.; Fuji, Y.; Yoshiyama, A.; Mashima, K., Sodium Methoxide: A Simple but Highly Efficient Catalyst for the Direct Amidation of Esters, Chem. Comm. 2012, 48, 5434-5436.

16 Caldwell, N.; Jamieson, C.; Simpson, I.; Tuttle, T., Organobase-Catalyzed Amidation of Esters with Amino Alcohols, Org. Lett. 2013, 15, 2506-2509.

17 MacMillan, D. S.; Murray, J.; Sneddon, H. F.; Jamieson, C.; Watson, A. J. B., Replacement of Dichloromethane within Chromatographic Purification: A Guide to Alternative Solvents, Green Chem. 2012, 14, 3016-3019.

18 MacMillan, D. S.; Murray, J.; Sneddon, H. F.; Jamieson, C.; Watson, A. J. B., Evaluation of Alternative Solvents in Common Amide Coupling Reactions: Replacement of Dichloromethane and N,N-Dimethylformamide, Green Chem. 2013, 15, 596-600.

19 McGonagle, F. I.; MacMillan, D. S.; Murray, J.; Sneddon, H. F.; Jamieson, C.; Watson, A. J. B., Development of a Solvent Selection Guide for Aldehyde-Based Direct Reductive Amination Processes, Green Chem. 2013, 15, 1159-1165.

20 Schwesinger, R. Chimia 1985, 39, 269.

21 Adams, J. P.; Alder, C. M.; Andrews, I.; Bullion, A. M.; Campbell-Crawford, M.; Darcy, M. G.; Hayler, J. D.; Henderson, R. K.; Oare, C. A.; Pendrak, I.; Redman, A. M.; Shuster, L. E.; Sneddon, H. F.; Walker, M. D., Development of GSK's Reagent Guides - Embedding Sustainability into Reagent Selection, Green Chem. 2013, 15, 1542-1549.

22 Frondel, C., Constitution and Polymorphism of the Pyroaurite and Sjogrenite Groups, American Mineralogist 1941, 26, 295-315. 
23 Jin, T.; Zhang, S.; Li, T., Transesterification of $\beta$-Ketoesters with Alcohols Catalyzed by Montmorillonite K-10, Green Chem. 2002, 4, 32-34.

24 Constable, D. J. C.; Jimenez-Gonzalez, C.; Henderson, R. K., Perspective of Solvent Use in the Pharmaceutical Industry, Org. Process Res. Dev. 2007, 11, 133-137.

25 Henderson, R. K.; Jimenez-Gonzalez, C.; Constable, D. J. C.; Alston, S. R.; Inglis, G. G. A.; Fisher, G.; Sherwood, J.; Binks, S. P.; Curzons, A. D., Expanding GSK's Solvent Selection Guide - Embedding Sustainability into Solvent Selection Starting at Medicinal Chemistry, Green Chem. 2011, 13, 854-862.

26 Elgue, S.; Prat, L.; Cabassund, M.; Cezerak, J., Optimisation of Solvent Replacement Procedures According to Economic and Environmental Criteria, Chem. Eng. J. 2006, 117, 169177.

27 Gani, R., Chemical Product Design: Challenges and Opportunities, Comput. Chem. Eng. 2004, 28, 2441-2457.

28 Antonucci, V.; Coleman, J.; Ferry, J. B.; Johnson, N.; Mathe, M.; Scott, J. P.; Xu, J., Toxicological Assessment of 2-Methyltetrahydrofuran and Cyclopentyl Methyl Ether in Support of Their Use in Pharmaceutical Chemical Process Development, Org. Process Res. Dev. 2011, $15,939-941$.

29 Watanabe, K.; Yamagiwa, N.; Torisawa, Y., Cyclopentyl Methyl Ether as a New and Alternative Process Solvent, Org. Process Res. Dev. 2007, 11, 251-258

30 http://www.zeon.co.jp. 
31 Winterberg, M.; Schulte-Korne, E.; Peters, U.; Nierlich, F. Methyl tert-Butyl Ether in Ullmann's Encyclopedia of Industrial Chemistry, Wiley-VCH: Hoboken, New Jersey, 2010

32 http://www.epa.gov/mtbe

33 Grundy, R. D. Strategies for Control of Man-Made Eutrophication, Environ. Sci. Technol. 1971, $5,1185-1190$

34 Carlson, R. Design and Optimization in Organic Synthesis; Elsevier: Amsterdam, 1992; Chapter 2. 


\section{Development of a Sustainable Catalytic Ester}

\section{Amidation Process}

Nicola Caldwell, ${ }^{\dagger}$ Craig Jamieson, ${ }^{*}{ }^{\dagger}$ Iain Simpson ${ }^{\ddagger}$ and Allan J. B. Watson ${ }^{\dagger}$

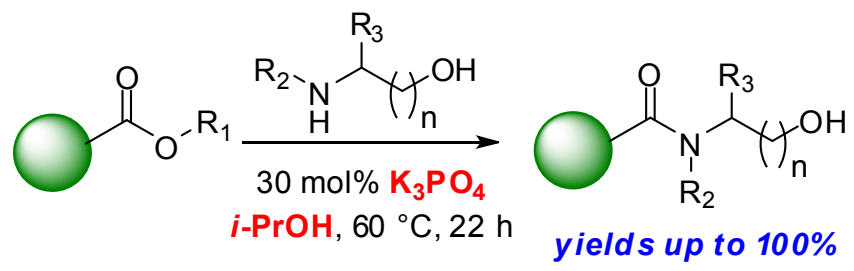

We report the development of a catalytic and sustainable amidation process enabling conversion of unactivated ester derivatives into amido-alcohol products. 